\title{
Influence of Layer Removal Methods in Residual Stress Profiling of a Shot Peened Steel using X-ray Diffraction
}

\author{
Rasha Alkaisee ${ }^{1, a}$ and Ru Lin Peng ${ }^{2, b}$ \\ 'Volvo Group Trucks Technology, Göteborg, Sweden \\ ${ }^{2}$ Department of Management and Engineering, Linköping University, Linköping, Sweden \\ arasha.alkaisee@volvo.com, bru.peng@liu.se
}

Key words: Residual stress profiling, XRD, Layer removal, Electro-polishing, Chemical etching

\begin{abstract}
For X-ray diffraction measurement of depth profiles of residual stress, step-wise removal of materials has to be done to expose the underneath layers to the X-rays. This paper investigates the influence of layer removal methods, including electro-polishing in two different electrolytes and chemical etching, on the accuracy of residual stress measurement. Measurements on two shotpeened steels revealed large discrepancy in subsurface distributions of residual stress obtained with the respective methods. Especially, the chemical etching yielded much lower subsurface compressive stresses than the electro-polishing using a so called AII electrolyte. The difference was explained by the influence of the different layer removal methods on the microscopic roughness.
\end{abstract}

\section{Introduction}

The X-ray diffraction technique for residual stress evaluation is well established and used widely in both research institutes and industrial laboratories. Because of the small penetration depth of X-ray beams in polycrystalline materials, the stress component perpendicular to the surface can often be considered to be approximate to zero. This assumption permits the in-plane surface stress components to be conveniently assessed by the standard $\sin ^{2} \psi$ method [1]. To determine a residual stress profile, material has to be taken away to expose the desired depth to the X-rays. The removal of material can lead to local relaxation of residual stresses and mathematical models are available to correct this effect for samples of simple geometry [2]. For the experimental method to be used for removing material, electrolytic polishing, likely the most commonly used technique, is considered to be suitable as it does not cause additional residual stresses [3]. Electro-polishing is carried out in an electrochemical cell. A DC current is applied via a built-in cathode in a shape of a pencil placed on the surface required to be polished out and a connection to the sample is established with a magnet or clamp on the sample, turning the surface into anodic. Material is removed through dissolution of the surface in the electrolyte. With suitable polishing parameters, a mirror like surface can be obtained. Other layer removal methods, such as chemical etching, are also used. In such methods, material is essentially etched away by an acid and a current or by an acid only. The choice of layer removal methods often depends on the environmental regulations of the countries where the companies are.

This paper investigates the influence of layer removal methods on the accuracy of depth profiling of residual stress. The study focused on electro-polishing in a commonly used electrolyte, AII, and chemical etching using a nitric acid but some results from electro-polishing in a salt solution were also included. Subsurface distributions of residual stress measured in two shot peened steels were compared and discussed with regards to microscopic roughness of the obtained surfaces.

\section{Experimental Details}

\subsection{Material and samples}

High strength low alloyed steel with ferritic-pearlitic microstructure and leaf spring steel with tempered martensite were used in the current studies. The former is denoted as steel A and the later steel B. Their chemical compositions are given in Table 1. Cylindrical specimen of 40mm in 
diameter made from steel $\mathrm{A}$ and flat specimen of dimension $90 \times 100 \times 26 \mathrm{~mm}^{3}$ made from steel $\mathrm{B}$ were shot-peened in different intensities by suppliers to induce a compressive residual stress field in the surface region. Fig. 1 shows the surface topography in steel A after shot-peening.

Table 1 Chemical composition of the steels

\begin{tabular}{|c|c|c|c|c|c|c|c|c|c|c|c|c|c|}
\hline Steel & $\mathrm{C}$ & $\mathrm{Si}$ & $\mathrm{Mn}$ & $\mathrm{P}$ & $\mathrm{S}$ & $\mathrm{Cr}$ & $\mathrm{Ni}$ & $\mathrm{Mo}$ & $\mathrm{Cu}$ & $\mathrm{Ti}$ & $\mathrm{V}$ & $\mathrm{Al}$ & $\mathrm{N}$ \\
\hline $\mathrm{A}$ & 0.43 & 0.57 & 1.46 & 0.018 & 0.04 & 0.21 & 0.16 & 0.14 & 0.17 & 0.021 & 0.09 & 0.019 & 0.015 \\
\hline $\mathrm{B}$ & 0.53 & 0.31 & 1.02 & 0.008 & $<0.005$ & 1.13 & 0.06 & 0.01 & 0.08 & - & 0.14 & 0.018 & 0.006 \\
\hline
\end{tabular}

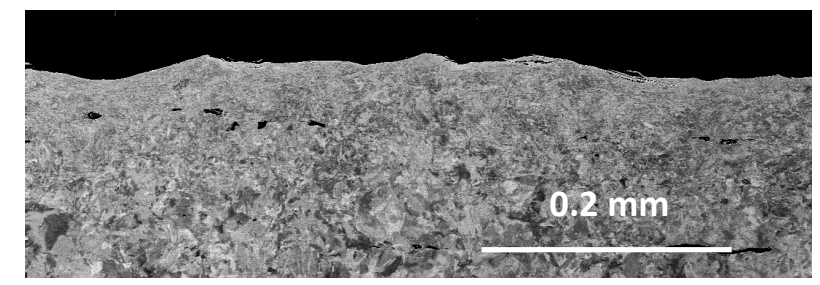

Fig. 1 ECC image showing the cross-section of the shot peened steel A.

\subsection{Method of layer removal and surface topographic analysis}

Three methods, electro-polishing using electrolyte AII (EP), chemical etching (CE) and electropolishing using a salt solution (SS) were included in the investigation. AII which consists of Perchloric acid is a commonly used electrolyte. It can give good polishing results on a number of metallic materials including steels and nickel based alloys. However, the Perchloric acid is considered to be hazardous to the health of the operator and environment $[3,4]$. The salt solution for electro-polishing is only salt (15\%) mixed with distilled water (according to an old recipe). For the chemical etching, a nitric acid (50 volume $\%$ in distilled water) was used. These methods were employed separately to remove material for stress determination at different depths of the subsurface layer. The applied voltage for EP in AII is $27 \mathrm{~V}$ and the area of polishing is approximately $9 \mathrm{~mm}$ in diameter. The area for CE and SS is about $5 \times 5 \mathrm{~mm}^{2}$.

\subsection{XRD measurement}

Residual stress measurements were carried out on a Stresstech X3000 G3 diffractometer equipped with $\mathrm{Cr}$ X-ray tube and position sensitive detectors. The collimator size is $2 \mathrm{~mm}$. Residual stresses were derived from measurements on the Fe-211 reflection using the standard $\sin ^{2} \psi$ method [1]with 6 to $7 \psi$-angles.

\section{Results and Discussion}

For XRD residual stress profiling by EP or CE, residual stress components in three in-plane directions, namely axial direction, $45^{\circ}$ to the axial direction and transverse direction, were obtained in two different locations on the same specimen, denoted as locations 1 and 2. For SS, only axial and transverse measurements in one location were made. Difference between the in-plane stress components for the same depth at the same location using the same method is found to be small. In addition, the difference does not seem to be dependent on the layer removal method. To simplify the analysis, the stress components are averaged for each measurement depth such that only one residual stress profile from each measurement location is used for comparison. The results for both residual stresses and FWHM are summarized in Fig. 2. The error bars are standard deviations of the mean values, which are rather small especially for the FWHM values. As can be seen, all the employed layer removal methods reveal compressive residual stresses in the subsurface. Furthermore, similar plastic deformation gradient, shown by the FWHM distributions, are also found for steel A. However, substantial difference in the residual stress distribution is observed. For steel A, the EP method, as shown by Fig. 2 (a), gives the highest compressive residual stresses and a small subsurface stress peak. The CE method yields the lowest compressive residual stresses which decrease monotonically with increasing depth. The stress distribution obtained by SS has 
intermediate stress values. Similar discrepancy between EP and CE is also detected for steel B (Fig. 2(b)). It can be concluded that the $\mathrm{CE}$ method gives much lower subsurface residual stresses for both steels in this study. This can be ascribed to local relaxation of residual stresses related to the etching process as discussed below.
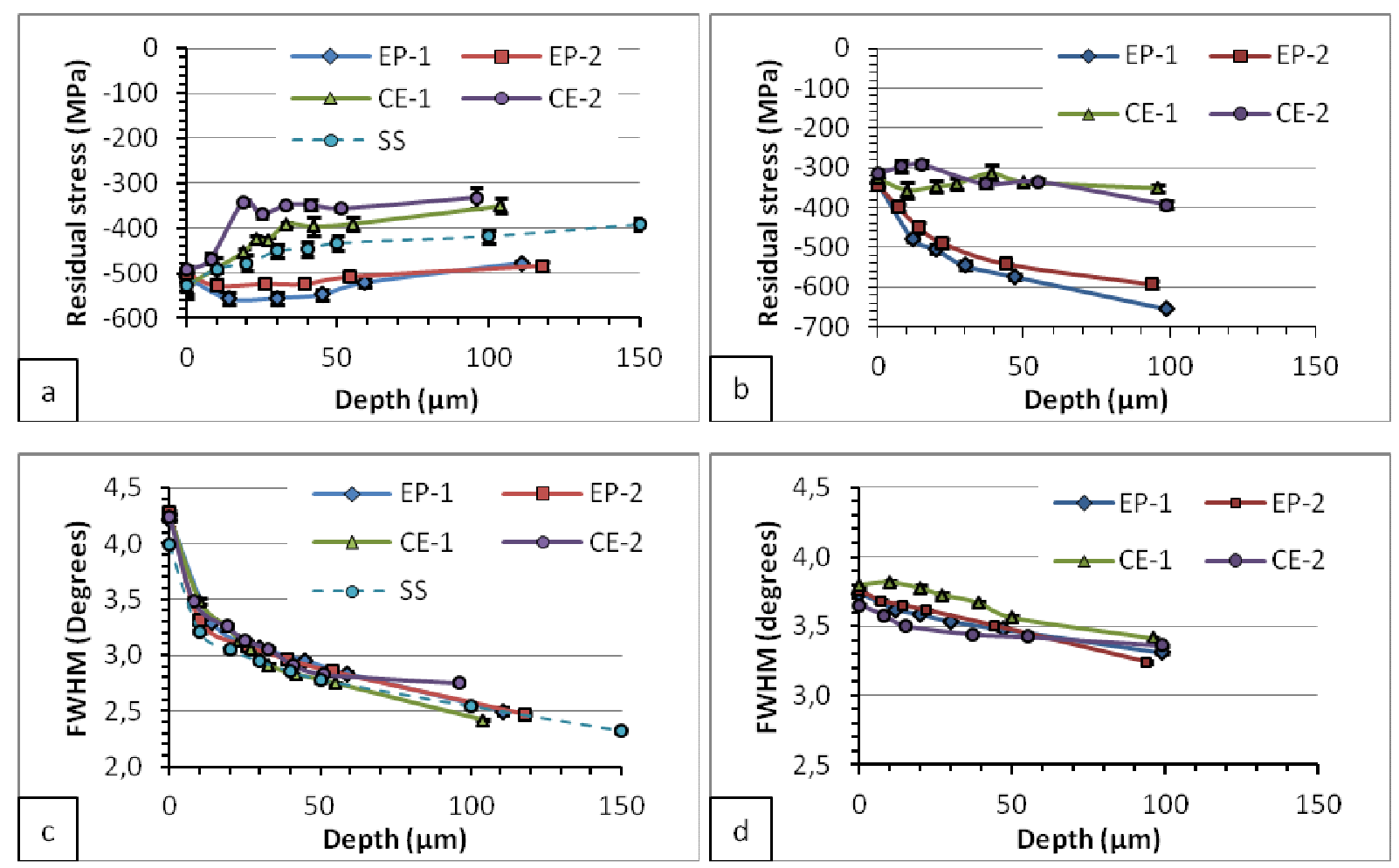

Fig. 2 Near surface residual stress and FWHM distributions in (a) and (c) for steel A and (b) and (d) for steel B. EP and CE refer to depth profiling using electrolytic polishing and chemical etching, respectively and SS is for solution etching. Numbers 1 and 2 in the key indicate the measurement locations 1 and 2 .

The surface topographic measurements (Fig. 3) and cross-section study of the polished/etched regions (Figs. 4 and 5) seem to provide explanations to the XRD results. Figs. 3(a) and (c) reveal a smooth surface with macroscopic (sub-millimeter) surface profile for the EP surfaces. As such a macroscopic roughness is also found in the CE surfaces (Figs. (b) and (d)), it is likely caused by the inherent chemical inhomogeneity of the steel. Figs. 3(b) and (d) also disclose much higher microscopic roughness with dense peaks and valleys in the CE surfaces. In agreement with the surface topographic measurements, the SEM observation in Fig. 4(a) shows a slightly curved but rather smooth surface in the EP samples. Surface roughness related to shot peening disappears completely. Under high magnifications (Figs. 4(b) and (c)), it can be seen that the EP process works uniformly over the surface and the polishing is essentially insensitive to grain orientations or boundaries between pearlitic colonies or between ferrite and pearlite. Phase dependent polishing does occur on certain pearlitic lamellar inclined to the surface, leaving a saw tooth feature at submicron scale (Fig. 4(b)). As the roughness is much smaller than the several micrometer penetration depth of X-rays in steel, such preferred polishing has little effect on the measured residual stress values. 
(a)

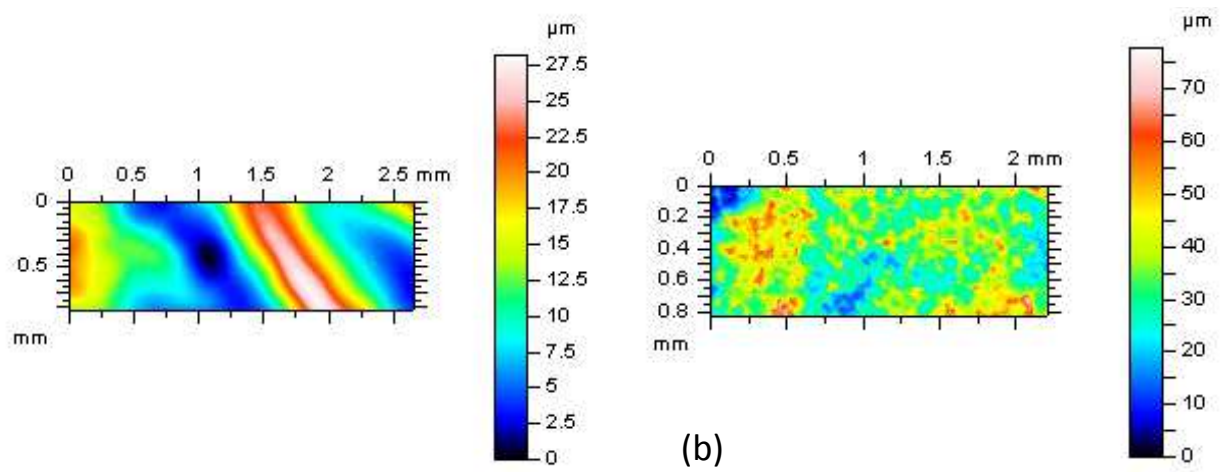

(c)

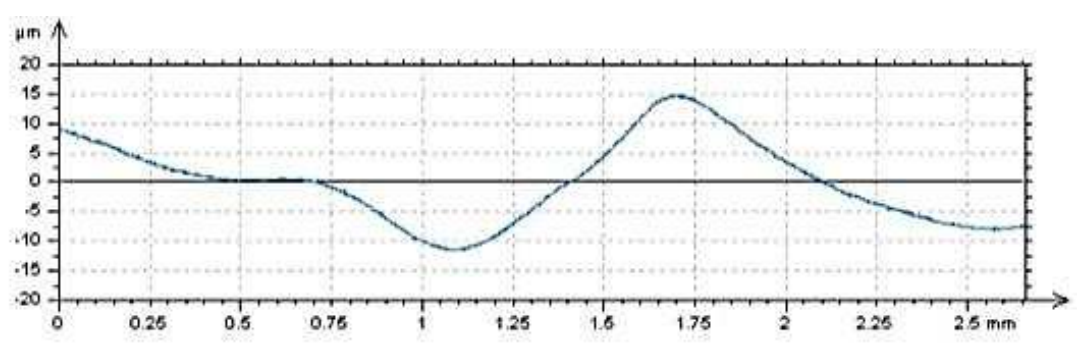

(d)

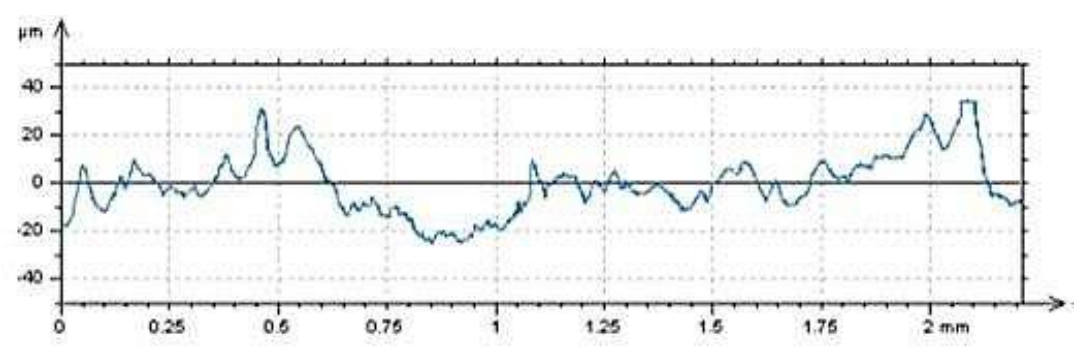

Fig. 3 Contour maps showing measured surface roughness (height profile) in steel A, (a) electropolished to a depth of $111 \mu \mathrm{m}$ and (b) chemically etched to a depth of $104 \mu \mathrm{m}$. (c) a horizontal line profile from (a) and (d) a line profile from (b). Ra is $0.935 \mu \mathrm{m}$ after electrolytic polishing and 2.313 $\mu \mathrm{m}$ after chemical etching.

In contrast to the EP surface, Figs. 4(d)-(f) and Fig. 5 show that besides the macroscopic (submillimeter) roughness like in the EP surface, $\mathrm{CE}$ also induces microscopic defects, resulting in local relaxation of residual stresses. The process is sensitive to the microstructure of the steel. The rate of etching differs between the different microstructure constituents and also depends on their orientations (Figs. 4(e) and (f)). The resulting microscopic roughness lowers the constraint between the microstructural constituents and thus relaxes partially the in-plane residual stresses. Etching also causes the formation of other defects, such as etching pits and preferred etching of microconstituent boundaries which appear as boundary cracks in Fig. 5. The formation of etching pits and especially the deep cracks along micro-constituent boundaries can further relax the in-plane residual stresses. Finally, the CE surface does not have a well-defined metallic surface as the EP surface does; a thin layer containing corrosion products and residual microstructure due to preferred etching is attached to the surface. Such a layer may not have a significant amount of ferrite which can contribute to the XRD residual stress measurement. However, it decreases the penetration depth by attenuating the X-ray beam. As the XRD measurement becomes then more superficial, the result of which shows an enhanced effect of surface defects from etching, namely lower residual stresses. 


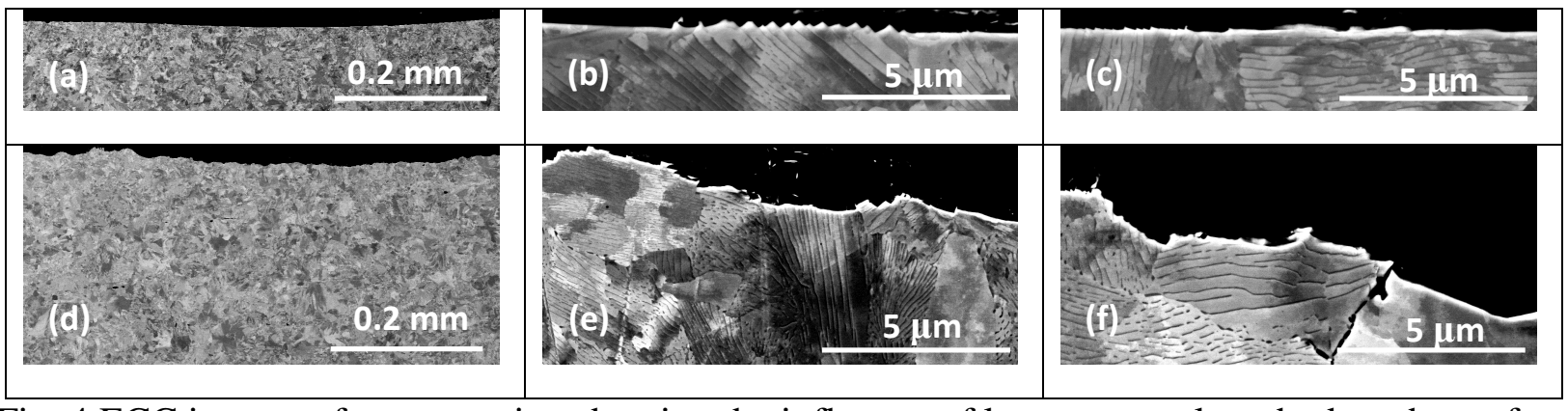

Fig. 4 ECC images of cross-section showing the influence of layer removal method on the surface microstructure for EP (a) - (c) and for CE (d) - (f) in steel A.
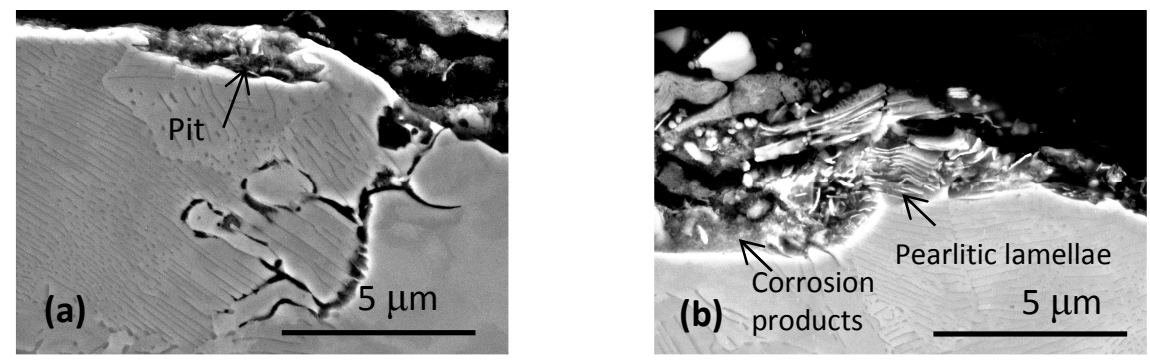

Fig. 5 Secondary electron images of cross-section showing corrosion pits and other surface heterogeneity in the chemically etched surface in steel A.

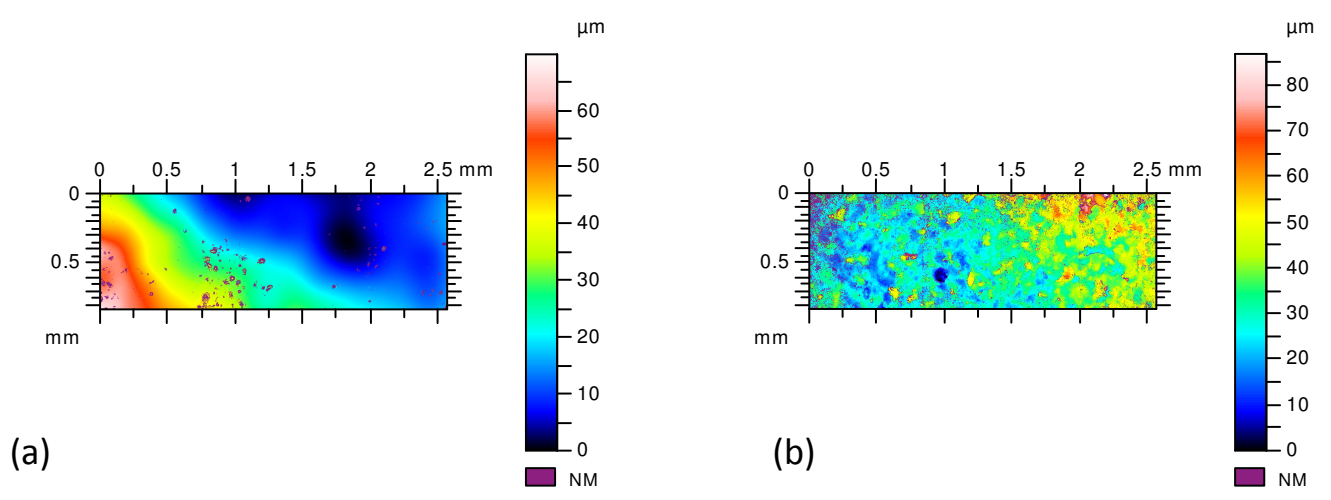

(a)

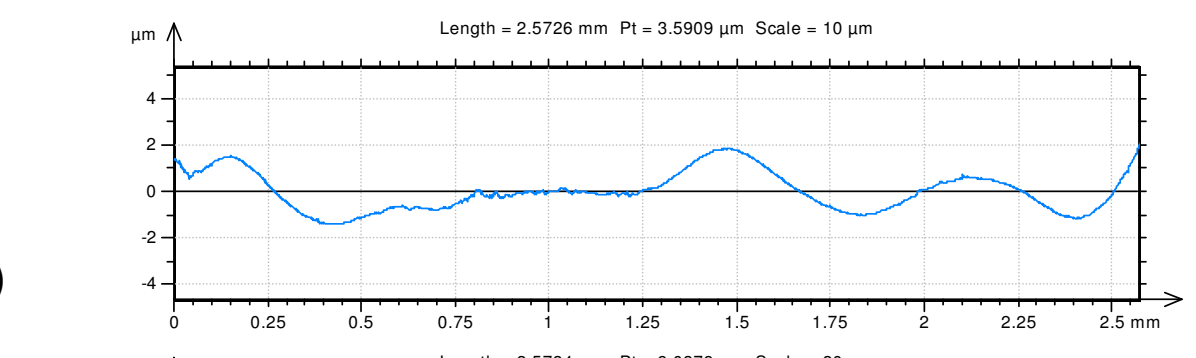

(c)

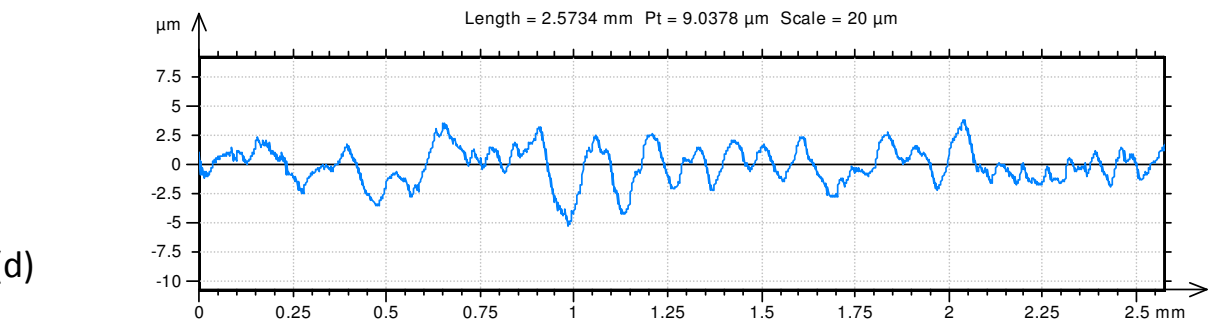

Fig. 6 Contour maps showing measured surface roughness (height profile) for steel B after electropolishing to a depth of $99 \mu \mathrm{m}$ (a) and after chemical etching to a depth of $96 \mu \mathrm{m}$. (c) Shows a horizontal line profile from (a) and (d) a line profile from (b). Ra is $0.306 \mu \mathrm{m}$ after electrolytic polishing and $1.360 \mu \mathrm{m}$ after chemical etching. 
The difference between locations 1 and 2 shown in Fig. 2 can be related to uncertainties in XRD measurements, a non-uniform shot peening process, or a poor reproducibility of layer removal process. The difference found for the EP sample is comparable to the scatter in surface residual stress before polishing, indicating a uniform shot peened surface and a good reproducibility of the EP method. For the CE sample, the discrepancy is larger than the surface scatter, which is likely caused by different etching results at the two locations. The contour maps of surface roughness for steel B show similar features as for steel A. No cross-section observation is made. However, it is believed that the chemical etching induced surface profile with microscopic roughness and defects also is responsible for the difference between the two methods observed in Fig. 2(b).

\section{Conclusions}

Three methods for material removal, namely electro-polishing in AII (EP), chemical etching (CE) in nitric acid and electro-polishing in a salt solution (SS), were compared for their influence on XRD depth profiling of residual stress in two shot-peened steels with subsurface compressive residual stresses. The CE method yielded lower subsurface compressive residual stresses than the EP method. SS performed on one of the steels gave intermediate subsurface compressive residual stresses which were between the EP and CE methods. Surface topographic measurements at certain polished or etched depths and SEM study of cross-sections showed that while macroscopic roughness of sub-millimeter exist for both EP and CE surfaces, the CE surface also exhibits substantial microscopic surface roughness resulted from a microstructural sensitive etching process and etching defects. Local relaxation of residual stresses due to such microscopic roughness and the existence of corrosion products and residual material on the $\mathrm{CE}$ surface led to much lower subsurface residual stresses in the obtained residual stress profiles.

The current work shows that the choice of chemical or electrochemical polishing method is important for accurate measurement of residual stress depth profiles in steels. Among the three methods investigated here, the electro-polishing in solution AII which gives a fine quality of polished surface with good reproducibility seems to be the best method for layer removal. Although the investigation was done only on a high strength low alloyed steel and a leaf spring steel, the conclusion is likely valid on other steels with a similar microstructure of ferrite-pearlite or tempered martensite.

\section{Acknowledgement}

Helps from Mrs. Annethe Billenius with metallographic sample preparation and Faculty Grant AFM (Faculty Grant SFO-MAT-LiU\#2009-00971) at Linköping University are acknowledged. Thanks to Ulla Boman (Manager at Metallic Materials, Volvo GTT), M.Sc. Lina Schäfer and Ph.D. Aurélien Tricoire at Volvo GTT, Materials Technology Department for their valuable support.

\section{References}

[1] I.C. Noyan, J.B. Cohen, Residual Stress Measurement by Diffraction and Interpretation, (1987).

[2] Moore, M.G. and Evans, W.P., mathematical Correction for Stress in Removed Layers in X-Ray Diffraction Residual Stress Analysis, SAE Trans. 66 (1958).

[3] ASTM E1558-09, Standard Guide for Electrolytic Polishing of Metallographic Specimens, ASTM international 2009.

[4] World Health Organization, Laboratory biosafety manual. - 3rd ed., World Health Organization (ISBN 924154650 6) 2004. 